\title{
NEUROFEEDBACK EN FIBROMIALGIA PARA EL TRATAMIENTO DE LA DEPRESIÓN Y LA ANSIEDAD ${ }^{\text {d。 }}$
}

\section{Carlos Barbosa Torres ${ }^{1} \mathbb{D}$, Sixto Cubo Delgado ${ }^{\circledR}$, María Luisa Bermejo García ${ }^{\circledR}, \&$ Florencio Vicente Castro}

\section{Universidad de Extremadura, Badajoz, España.}

\begin{abstract}
RESUMEN
Este trabajo versa sobre neurofeedback en pacientes con fibromialgia. Este síndrome aglutina una gran cantidad de síntomas diversos, que abarcan desde el dolor crónico, fatiga y cansancio crónico, problemas atencionales, de memoria y de sueño. A pesar de que es un síndrome idiopático múltiples estudios apuntan a que su origen está relacionado con el Síndrome de Sensibilización Central (SSC). Como intervención realizamos un entrenamiento mediante neurofeedback cuyo propósito es mejorar las variables ansiedad y depresión en fibromialgia. Utilizamos un protocolo estandarizado de ritmos sensoriomotores (SMR) ya que el entrenamiento de estas ondas repercute en el sistema dopaminérgico que a su vez está relacionado con la facilitación de las emociones positivas. Los resultados muestran que el neurofeedback mejora los trastornos de depresión y ansiedad en fibromialgia.
\end{abstract}

Palabras Claves

fibromialgia, síndromes somáticos, señales eléctricas, sensibilización, ratio SMR/Theta

\begin{abstract}
In the present work we found a study on neurofeedback in patients with fibromyalgia syndrome. This syndrome brings together a large number of very diverse symptoms, ranging from chronic pain, chronic fatigue to sleep problems. Although it is an idiopathic syndrome, multiple studies suggest that its origin is related to the Central Sensitization Syndrome (SSC). We conducted a training through neurofeedback whose purpose is to improve the variables anxiety and depression. We use a standardized protocol of sensorimotor rhythms (SMR) that can improve the stability of the cerebral cortex, the training of these waves affect the dopaminergic system which in turn is related to the facilitation of positive emotions. The results show that neurofeedback improves significantly depression and anxiety disorders.
\end{abstract}

\section{Keywords}

fibromyalgia, somatic syndromes, electrical signals, sensitization, SMR/Theta ratio

\footnotetext{
${ }^{1}$ Correspondence about this article should be addressed to Carlos Barbosa Torres: carlosbt92@gmail.com
} 


\section{NEUROFEEDBACK IN FIBROMYALGIA FOR THE TREATMENT OF DEPRESSION AND ANXIETY}

\section{Introducción}

La fibromialgia es un síndrome generalizado asociado con múltiples síntomas que abarcan desde problemas del sueño, fatiga o cansancio crónico, alteraciones en la cognición y una gama amplia de síntomas somáticos. Su principal característica es dolor músculo-esquelético difuso, de origen no articular y crónico que se manifiesta en puntos corporales específicos (Garg \& Deodhar, 2012).

A pesar de las numerosas investigaciones que se han realizado sobre este trastorno, no se conoce la causa exacta. No hay ningún biomarcador ni prueba diagnóstica que la corrobore, por lo tanto, se realizaron acuerdos entre expertos para que su criterio diagnóstico se basará en unas evaluaciones de exámenes físicos, antecedentes sanitarios y pruebas de laboratorio que descarten el diagnóstico diferencial (Fitzcharle, Shir, Ablin, Buskila, Amital \& Henningsen, 2013; Fitzcharles, Ste-Marie, Goldenberg, Pereira, Abbey \& Choiniere, 2013).

La hipótesi más extendida actualmente, con respecto a la fibromialgia y demás síndromes similares son, es que se producen por un "síndrome de sensibilización central" (SSC) (Boomershine, 2015; Yunus, 2012). La argumentación principal es que el cerebro al experimentar el dolor, lo potencia y mantiene llegando a crear una situación permanente. Las técnicas actuales no han conseguido interrumpir el proceso de sensibilización. Las zonas que pueden presentan esta sensibilización son las zonas talámicas o del córtex sensorial, motivo por el cual el proceso también es llamado sensibilización central suprasegmental. Este mecanismo según investigaciones se encuentra detrás de otros síndromes somáticos o los síntomas dolorosos, así como en la depresión. La alteración de los mecanismos inhibitorios, en especial la desactivación de éstos, puede considerarse como un hecho que debemos tener en cuenta a la hora de explicar la patología (Stahl, 2012).

A pesar de que no se sabe con exactitud el origen de la fibromialgia, los cambios que se producen en el Sistema Nervioso Central tienen un papel muy importante en el desarrollo de la fibromialgia por lo que si se observan variaciones en los ritmos cerebrales que se repiten en todos los pacientes se pueden hallar evidencias de actividad neurofisiológica disfuncional en un grupo concreto y utilizar esa información para desarrollar un tratamiento (Menche, 2011). 
Una de las mayores ventajas que presenta el neurofeedback, ante otro tipo de tratamientos para tratar la fibromialgia es que permite intervenir directamente a nivel cortical reorganizando las señales eléctricas para conseguir una desensibilización central y proporcionando un alivio en los síntomas (Jensen, Hakimian, Sherlin, \& Fregni, 2008).

Kayiran, Dursun, Dursun, Ermutlu, y Karamürsel (2010) realizaron uno de los último y más recientes estudios con pacientes con fibromialgia, mediante el entrenamiento de los ritmos sensoriomotores para favorecer los mecanismos inhibitorios del sistema nervioso central. Se observó una disminución significativa en la relación Theta/SMR al final del tratamiento, en comparación con la línea base y la mejora en de los test de control de variables psicológicas.

Pavlenko, Chernyi y Goudkina (2009) realizaron estudios mediante el entrenamiento de los ritmos sensoriomotores (SMR) (12-15 hz) y propusieron que podría ser eficaz para la estabilidad emocional, ya que estos ritmos, tienen repercusión en el sistema dopaminérgico (DA) en el tegmentum ventral. La estabilidad emocional y el afecto positivo podrían modificarse mediante el entrenamiento de los ritmos sensoriomotores ya que esto repercute en el sistema dopaminérgico que a su vez está relacionado con la facilitación de las emociones positivas y el mantenimiento de las conductas de aproximación.

Según Sterman (2000), el entrenamiento de los SMR puede mejorar la estabilidad de la corteza. Sin ir más allá, las investigaciones sobre el entrenamiento mediante biofeedback para reducir el impulso simpático y, por ende, estabilizar el eje adrenalhipotalámico-límbico-cortical, ha dado muy buenos resultados. Los ritmos sensoriomotores se usan para la atención relajada y cuerpo tranquilo. Con el incremento de los SMR la persona se vuelve más relajada.

Por todos estos antecedentes se planeó el siguiente objetivo y las siguientes hipótesis:

\section{Objetivo}

Evaluar cómo el condicionamiento instrumental generado por el neurofeedback repercute en los trastornos psicológicos de depresión y ansiedad en pacientes con fibromialgia.

\section{Hipótesis}

Hipótesis 1: El neurofeedback (Protocolo SMR) mejora la depresión de los pacientes con fibromialgia. 
Hipótesis 2: Estudiar la ansiedad en relación con la aplicación del neurofeedback (Protocolo SMR) en pacientes con fibromialgia.

Contrastamos empíricamente esta hipótesis a través de las siguientes subhipótesis.

Hipótesis 2.1: El neurofeedback (Protocolo SMR) mejora la ansiedad estado de los pacientes con fibromialgia.

Hipótesis 2.2: El neurofeedback (Protocolo SMR) mejora la ansiedad rasgo de los pacientes con fibromialgia.

\section{Método}

\section{Participantes}

La muestra se calculó de una total de más de 150 pacientes, de los cuales aproximadamente $60 \%$ no superó los criterios de inclusión y exclusión, por lo que el estudio se realizó finalmente con 40 participantes remitidos de diversas asociaciones de fibromialgia de Extremadura (España), teniendo como principal característica su carácter no probabilístico. Del total de la muestra 36 fueron mujeres y 4 hombres, con una edad comprendida entre los 31 y los 70 años de los cuales 14 sujetos se encontraban entre 31/51 años, 23 entre los 51/65 años y 3 eran mayores de 65 años. El nivel educativo de los participantes resultó homogéneo 12 de educación infantil, 6 de educación primaria, 15 de COU/ESO, 15 de bachillerato y 7 con formación universitaria. De la totalidad de los sujetos 20 no han estado de baja médica, 7 se encontraban en el momento del estudio de baja médica y 13 habían estado de baja médica con anterioridad. La selección de la muestra estuvo marcada por los criterios de inclusión y exclusión.

Los criterios de inclusión:

(1) Tener entre 16 años o más. Si el paciente es menor deberá presentar la autorización de un tutor.

(2) Estar diagnosticado de fibromialgia bajo los criterios de la ACR (American College of Rheumatology).

No tener otros problemas de salud como problemas coronarios, diabetes mellitus, padecer o haber padecido cáncer o alcoholismo.

\section{Instrumentos}

La información se obtuvo mediante una entrevista con los participantes donde cumplimentaron los instrumentos seleccionados. 
El cuestionario utilizado para medir la depresión en este estudio es el BDI (Inventario de Depresión de Beck). La versión original la elaboró Beck en 1961 (Beck, Ward, Mendelson, Mock \& Erbaugh, 1961) y la primera adaptación al castellano fue realizada por Conde y Useros (1975). El inventario tiene como objetivo medir los estados de ánimo con el que se detecta la presencia de la depresión y se mide exactamente su gravedad mediante una escala de tipo Likert. Este cuestionario ha sido estudiado de manera muy exhaustiva, mostrando una alfa de Cronbach de 0.83 (Beck, Steer \& Garbin, 1988; Richter, Werner \& Heerlein, 1998).

El State-Trait Anxiety Inventory (STAI) es uno de los cuestionarios más utilizados para medir la ansiedad y fue construido y desarrollado por Spielberger, Gorsuch y Lushene (1970). El STAI Inventario de Ansiedad, evalúa dos dimensiones de ansiedad (rasgo y estado) mediante una escala de tipo Likert (Spielberg, Goursch \& Lushene, 1982), ambas escalas están diferenciadas tanto en su administración como en su evaluación. Miden dos conceptos independientes de la ansiedad como estado (E) y como rasgo (R). Basados en los componentes del modelo de ansiedad desarrollado por Spielberger. En la revisión de la versión española realizada por Guillén y Buela (2011), compuesta por 1.036 participantes españoles (352 eran hombres y 667 mujeres) con edades comprendidas entre 18 y 69 años obtuvo un análisis de fiabilidad significativo. Para los ítems de ansiedad rasgo obtuvo un alfa de Cronbach de 0.90 y la ansiedad estado alcanzó un alfa de .94.

Los instrumentos de recogida y análisis de las variables electrofisiológicas son productos de la empresa Thought Technology, una de las principales compañías fabricantes de productos de biofeedback y neurofeedback del mundo. Tiene certificado de dispositivos médicos para sus aparatos de biofeedback (Thought Technology, 2018).

Se utilizó el Codificador FlexComp Infiniti que es un dispositivo de ocho canales que se usa para el análisis psicofisiológico en tiempo real, biorretroalimentación y adquisición de datos. El codificador tiene una entrada específica en la cual conectamos un sensor de electroencefalograma. El software utilizado es el BioGraph Infiniti desarrollado para captar y analiza los datos, y para realizar el condicionamiento instrumental utilizamos un protocolo de ritmos sensoriomotores. 


\section{Procedimiento}

Se trata de un estudio experimental realizado gracias a la colaboración de varias asociaciones de fibromialgia de la provincia de Badajoz, Extremadura (España) y a la Unidad del Dolor de referencia ubicada en la misma comunidad, quienes nos ofrecieron asesoramiento profesional tanto para el acceso a la muestra, como para la selección de los criterios de inclusión. El tratamiento de neurofeedback fue aplicado 3 veces por semana en las propias asociaciones durante un total de 20 sesiones ( 7 semanas de tratamiento) cumpliendo todas las normas de seguridad y protocolos estandarizados para la aplicación de este tipo de terapias. Se utilizó un protocolo de ritmos sensoriomotores (SMR) estandarizado y desarrollado por Thought Technology para la realización del entrenamiento.

En cuanto a los aspectos éticos se han respetado todos los principios éticos universales que rigen la conducción de investigación en Psicología y la realización de este estudio ha sido estrictamente necesario respetar los siguientes preceptos éticos fundamentales, al igual que se expone que esta investigación carece de conflicto de intereses. Para la realización de este estudio los autores declaran que han seguido los protocolos de su centro de trabajo sobre la publicación de datos de pacientes. Han respetado el derecho a la privacidad y han seguido el Código de Nüremberg (1974), donde se especifica que es imprescindible el consentimiento voluntario de la persona.

\section{Análisis de datos}

El proceso seguido para realizar el análisis inferencial ha sido el siguiente:

1. Se presenta la tendencia de los resultados a través de una gráfica. En la abscisa se representa la variable en estudio, en la ordenada los resultados obtenidos en la serie de datos considerando los valores mínimo y máximo teóricos de la distribución.

2. Cuando la variable que actúa como dependiente es medida en una escala de intervalo o de razón se aplican las siguientes pruebas para decidir si es necesario utilizar una prueba paramétrica o no paramétrica:

a. Kolmogorov-Smirnov, para contrastar la hipótesis nula de que la distribución teórica en la población es normal.

b. Rachas, para contrastar la hipótesis nula de que la distribución teórica en la población es aleatoria. 
c. Levene, no se realizará para contrastar la hipótesis nula relacionada con la igualdad de las varianzas entre las diferentes variables analizadas debido a que son muestras relacionadas.

3. Se aplica el modelo estadístico necesario para contrastar la hipótesis de trabajo.

4. Se da respuesta al contraste realizado de la hipótesis nula y por tanto también de la hipótesis de trabajo.

5. Cuando se valora el impacto de una intervención, además de la significación estadística, se utiliza como criterio el análisis del tamaño del efecto, a través del coeficiente d de Cohen, que aparece en la tabla 3.

\section{Resultados}

A continuación, se observan los resultados de las variables psicológicas medidas en las tablas 1 y 2 .

Tabla 1

Resultados de las variables psicológicas

\begin{tabular}{lllllll}
\hline Depresión & Ausente & Leve & Intermitente & Moderada & Grave & Extrema \\
\hline Pre & $2(5 \%)$ & $7(17.5 \%)$ & $8(20 \%)$ & $15(37.5 \%)$ & $5(12.5 \%)$ & $3(7.5 \%)$ \\
Post & $7(17.5 \%$ & $15(37.5 \%)$ & $5(15.5 \%)$ & $9(22.5 \%)$ & $3(7.5 \%)$ & $1(2.5 \%)$ \\
\hline Ansiedad & \multicolumn{2}{c}{ Estado } & & \multicolumn{2}{c}{ Rasgo } & \\
\hline \multicolumn{2}{c}{$\leq 5$} & $>5$ & & 55 & $>5$ \\
Pre & $4(10 \%)$ & $36(90 \%)$ & & $7(17.5 \%)$ & $33(82.5 \%)$ & \\
Post & $7(17.5 \%)$ & $33(82.5 \%)$ & & $11(27.5 \%)$ & $29(72.5 \%)$ & \\
\hline
\end{tabular}

Tabla 2

Resultados de las medias totales de las variables psicológicas

\begin{tabular}{llll}
\hline & Depresión & Ansiedad Estado & Ansiedad Rasgo \\
\hline Media total PRE & 23,88 & 7,4 & 7,23 \\
Media total POST & 18,1 & 6,5 & 6,5 \\
\hline
\end{tabular}

A continuación, se observa una tabla (tabla 3 ) con las hipótesis analizadas, donde se muestra el grado de significación y la d de cohen. Así como la figura 1 que marca la tendencia de las medidas totales de los cuestionarios de depresión y ansiedad, y la figura 2 que muestra las puntuaciones de las variables psicológicas por los rangos propios de cada cuestionario. 
Tabla 3

Hipótesis contrastadas

\begin{tabular}{|c|c|c|c|c|}
\hline \multicolumn{5}{|c|}{ Hipótesis 1: El neurofeedback (Protocolo SMR) mejora la depresión de los pacientes con fibromialgia } \\
\hline Hipótesis & Estadístico & (p) & $\begin{array}{c}\text { d de } \\
\text { Cohen }\end{array}$ & Resultado \\
\hline $\begin{array}{l}\text { Hipótesis 1: } \text { El neurofeedback } \\
\text { (Protocolo SMR) mejora la depresión de } \\
\text { los pacientes con fibromialgia }\end{array}$ & $\begin{array}{c}\text { Tde } \\
\text { Wilcoxon }\end{array}$ & 000 &, 584 & $\begin{array}{l}\text { Rechazamos la hipótesis nula } \\
(, 000<0.05) \text {. Aceptamos la } \\
\text { hipótesis de trabajo. }\end{array}$ \\
\hline \multicolumn{5}{|c|}{$\begin{array}{l}\text { Hipótesis 2: Estudiar la ansiedad en relación con la aplicación del neurofeedback (Protocolo SMR) en } \\
\text { pacientes con fibromialgia }\end{array}$} \\
\hline Hipótesis & Estadístico & (p) & $\begin{array}{c}\text { d de } \\
\text { Cohen }\end{array}$ & Resultado \\
\hline $\begin{array}{l}\text { Hipótesis 2.1: El neurofeedback } \\
\text { (Protocolo SMR) mejora la ansiedad } \\
\text { estado de los pacientes con fibromialgia. }\end{array}$ & $\begin{array}{c}\text { Tde } \\
\text { Wilcoxon }\end{array}$ &, 000 &, 565 & $\begin{array}{l}\text { Rechazamos la hipótesis nula } \\
(, 000<0.05) \text {. Aceptamos la } \\
\text { hipótesis de trabajo. }\end{array}$ \\
\hline $\begin{array}{lll}\text { Hipótesis } & 2.2: & \text { El neurofeedback } \\
\text { (Protocolo } & \text { SMR) } & \text { mejora la ansiedad }\end{array}$ & $\begin{array}{l}\text { T de } \\
\text { Wilcoxon }\end{array}$ & 006, & ,431 & $\begin{array}{l}\text { Rechazamos la hipótesis nula } \\
(, 000<0.05) \text {. Aceptamos la } \\
\text { hipótesis de trabajo. }\end{array}$ \\
\hline
\end{tabular}

* Correlación significativa para $\mathrm{p}<.05$

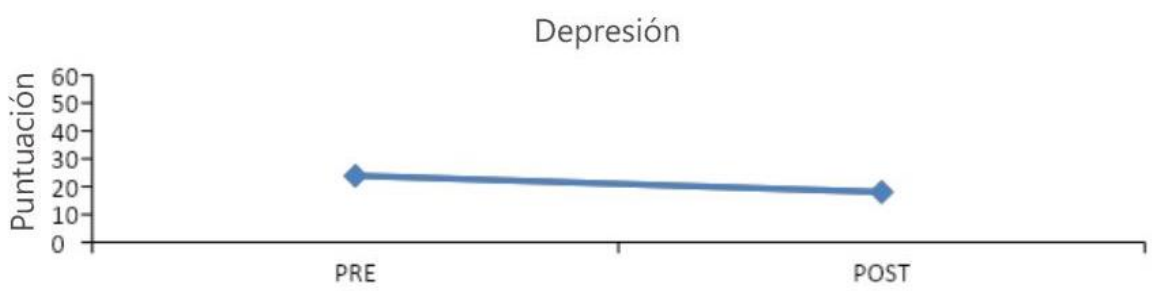

Ansiedad Estado

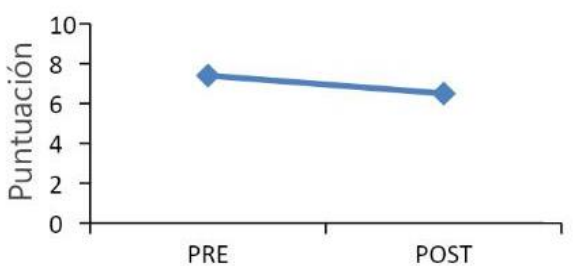

Ansiedad Rasgo

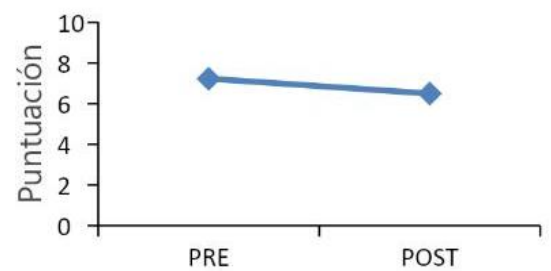

Figura 1: Resultados de las medias totales PRE/POST de las variables medias en los cuestionarios depresión y ansiedad estado y rasgo. En la parte superior las medidas PRE/POST del cuestionario Beck Depression Inventory. En la parte inferior las medias del cuestionario State-Trait Anxiety Inventory. En la parte izquierda las medidas PRE/POST en ansiedad estado y en la parte inferior derecha la medida PRE/POST en ansiedad rasgo.
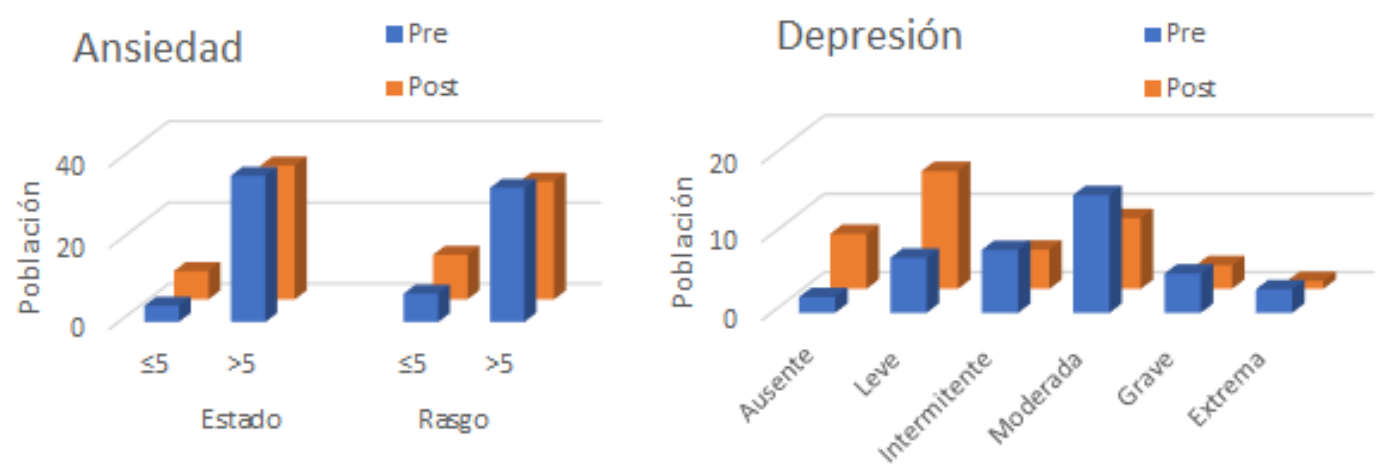

Figura 2: Resultado de las puntuaciones de los cuestionarios de ansiedad y depresión por los rangos propios de cada cuestionario. En la parte izquierda las puntuaciones del cuestionario State-Trait Anxiety Inventory, y en la parte derecha las puntuaciones del Beck Depression Inventory. 


\section{Discusión}

En relación con el objetivo principal de esta investigación, que es evaluar cómo el condicionamiento instrumental generado por el neurofeedback repercute en los trastornos psicológicos de depresión y ansiedad en pacientes con fibromialgia, se aprecia que hay una mejoría de las variables, depresión, ansiedad. Esta mejoría en las variables puede estar explicada por el aprendizaje que presentan los participantes y su capacidad de adaptarse a la exigencia de la terapia de neurofeedback.

Son múltiples los estudios que muestran la incidencia de los trastornos depresivos y de ansiedad en pacientes con fibromialgia (Homannet, Stefanello, Góes, Breda, Paiva \& Leite, 2012; Goldenberg, 2010; Falcão, Sales, Leite, Feldman, Valim \& Natour, 2008) pero apenas hay estudios cuya variable independiente sea el tratamiento de neurofeedback, y mucho más concreto, mediante un protocolo de SMR en pacientes con fibromialgia (Kayiran et al, 2010; Pavlenko et at, 2009). En nuestro estudio, al igual que es de Kayiran et al. 2010, se hace consciente la mejora de la depresión y ansiedad como variable psicológica. Según los objetivos descritos en el trabajo de investigación, se expone que el entrenamiento mediante la técnica de neurofeedback ha provocado un cambio en los patrones eléctricos cerebrales, especialmente si se atiende al moldeamiento que ha sufrido el ratio SMR/Theta. El moldeamiento de las ondas cerebrales es un proceso complejo de entender y entrenar, motivo por el cual los pacientes presentan cambios lentos y no siempre constantes. En esta investigación, los pacientes con fibromialgia se han adaptado de forma adecuada a los requisitos que el entrenador ha marcado en el software utilizado para la investigación. El esfuerzo cognitivo necesario que han realizado los pacientes con fibromialgia para moldear su electroencefalograma se debe a la terapia de neurofeedback, y según lo expuesto por Stahl (2012), la activación de los procesos inhibitorio podría ser beneficioso para reducir la estimulación global en este tipo de pacientes.

Este estudio muestra que aumento del ratio SMR/Theta contribuye a mantener un equilibrio en las funciones cerebrales, como ya expuso Sterman (2000), lo cual permite que los pacientes con fibromialgia atiendan mejor a los estímulos externos. El aumento del ratio, facilita y potencia los mecanismos inhibitorios del sistema nervioso central y, como resultado, la información sensorial que llega al cerebro puede filtrarse y clasificarse mejor. Como resultado se obtiene un sistema nervioso menos saturado de estímulos y con 
mayor capacidad de procesamiento, debido a esto el porcentaje de pacientes que al inicio del estudio presentaban un diagnóstico depresivo era del $60.5 \%$ y tras la aplicación de la terapia, es un $32.5 \%$. En cuanto a los pacientes con ansiedad-estado un $90 \%$ presentaban más de 5 en puntuaciones decatipo y tras la aplicación del neurofeedback, este porcentaje disminuyó a un $82.5 \%$. En cuanto a la ansiedad-rasgo un $82.5 \%$ presentaban más de 5 en puntuaciones decatipo y tras la aplicación del neurofeedback, este porcentaje disminuyó a un $72.5 \%$. Si se observa el valor la d de Cohen en las hipótesis se observa que presentan un valor medio, lo que indica que la diferencia entre el grupo antes y después del tratamiento de neurofeedback presentan un tamaño del efecto medio. Estos resultados van en consonancia con los estudios de Pavlenko et al. (2009), que también describe el aumento en la estabilidad emocional general de los pacientes con fibromialgia tras la aplicación de la terapia de neurofeedback mediante el entrenamiento de los SMR.

Según las hipótesis planteadas y los resultados expuestos, se concluye que el neurofeedback mejora significativamente los trastornos de depresión y ansiedad en pacientes con fibromialgia por lo que podría ser una posible solución a los problemas planteados por estos pacientes. 


\section{Referencias}

Beck, A. T., Ward, C. H., Mendelson, M., Mock, J., \& Erbaugh, J. (1961). An inventory for measuring depression. Arch Gen Psychiatry, 4, 561-71.

Beck, A., Steer, R., \& Carbin, M. (1988). Psychometric properties of the Beck Depression Inventory: Twenty-five years of evaluation. Clinical Psychology Review, 8(1), 77100. http://dx.doi.org/10.1016/0272-7358(88)90050-5

Boomershine, C. S. (2015). Fibromyalgia: the prototypical central sensitivity syndrome. Current Rheumatology Reviews, 11(2), 131-145.

Conde, V., \& Useros, E. (1975). Adaptación castellana de la escala de evaluación conductual para la depresión de Beck. Rev Psiquiatr Psicol Med Eur Am, 12(21736).

Egner, T., \& Gruzelier, J. (2001). Learned self-regulation of EEG frequency components affects attention and event-related brain potentials in humans. Neuroreport, 12(18), 4155-4159. http://dx.doi.org/10.1097/00001756-200112210-00058

Falcão, D. M., Sales, L., Leite, J., Feldman, D., Valim, V., Natour, J. (2008). Cognitive behavioral therapy for the treatment of fibromyalgia syndrome: a randomized controlled trial. J Musculoskelet Pain, 16(3),133-40.

Fitzcharles, M., Shir, Y., Ablin, J., Buskila, D., Amital, H., Henningsen, P., \& Häuser, W. (2013). Classification and Clinical Diagnosis of Fibromyalgia Syndrome: Recommendations of Recent Evidence-Based Interdisciplinary Guidelines. Evidence-Based Complementary And Alternative Medicine, 1-9. http://dx.doi.org/10.1155/2013/528952

Fitzcharles, M., Ste-Marie, P., Goldenberg, D., Pereira, J., Abbey, S., \& Choinière, M. et al. (2013). 2012 Canadian Guidelines for the Diagnosis and Management of Fibromyalgia Syndrome: Executive Summary. Pain Research and Management, 18(3), 119-126. http://dx.doi.org/10.1155/2013/918216

Garg, N. \& Deodhar, A. (2012). New and modified fibromyalgia diagnostic criteria: Ambiguity, uncertainty, and difficulties complicate diagnosis and management. $J$ Musculoskel Med, 29, 1-5.

Goldenberg, D. L. (2002). The interface of pain and mood disturbances in the rheumatic diseases. Semin Arthritis Rheum, 40(1),15-31.

Guillén, A. \& Buela, G. (2011). Actualización psicométrica y funcionamiento diferencial de los ítems en el State Trait Anxiety Inventory (STAI). Psicothema, 23(3), 510515.

Homann, D., Stefanello, J. M. F., Góes, S. M., Breda, C. A., Paiva, E. S., Leite, N. (2012). Percepção de estresse e sintomas depressivos: funcionalidade e impacto na qualidade de vida em mulheres com fibromialgia. Rev Bras Reumatol, 52(3), 32430.

Jensen, M., Hakimian, S., Sherlin, L., \& Fregni, F. (2008). New Insights Into Neuromodulatory Approaches for the Treatment of Pain. The Journal Of Pain, 9(3), 193-199. http://dx.doi.org/10.1016/j.jpain.2007.11.003

Kayıran, S., Dursun, E., Dursun, N., Ermutlu, N., \& Karamürsel, S. (2010). Neurofeedback Intervention in Fibromyalgia Syndrome; a Randomized, Controlled, Rater Blind Clinical Trial. Applied Psychophysiology And Biofeedback, 35(4), 293-302. http://dx.doi.org/10.1007/s10484-010-9135-9

Menche, D. S. (2001). Correlation between osteoarthritic cartilage damage and levels of proteinases and proteinase inhibitors in synovial fluid from the knee joint. Arthroscopy, 17(7), 790. 
Pavlenko, V., Chernyi, S., \& Goubkina, D. (2009). EEG Correlates of Anxiety and Emotional Stability in Adult Healthy Subjects. Neurophysiology, 41(5), 337-345. http://dx.doi.org/10.1007/s11062-010-9111-2

Richter P., Werner J. \& Heerlein, A. (1998). On the validity of the Beck Depression Inventory. A review. Psychopathology, 31, 160-168.

Spielberger C.D., Gorsuch, R.L. \& Lushene, R.E. (1982). Cuestionario de Ansiedad Estado/Rasgo. Madrid: TEA.

Spielberger, C. D., Gorsuch, R. L. \& Lushene, R. E. (1970). STAI Manual for the StateTrait Anxiety Inventory (Sef-Evaluation Questionnaire). Palo Alto California: Consulting Psychologists Press.

Stahl, S. M. (2013). Stahl's Essential Psychopharmacology: Neuroscientific Basis and Practical Applications. Cambridge, UK: Cambridge University Press.

Sterman, M. (2000). Basic Concepts and Clinical Findings in the Treatment of Seizure Disorders with EEG Operant Conditioning. Clinical EEG And Neuroscience, 31(1), 45-55. http://dx.doi.org/10.1177/155005940003100111

Thoughttechnology (2018). Recuperado de http://thoughttechnology.com/

Yunus, M. (2012). The Prevalence of Fibromyalgia in Other Chronic Pain Conditions. Pain Research And Treatment, 2012, 1-8. http://dx.doi.org/10.1155/2012/584573 